\title{
Note on strongly hyperbolic systems with involutive characteristics
}

\author{
Guy Métivier * and Tatsuo Nishitani ${ }^{\dagger}$
}

August 4, 2016

\begin{abstract}
We consider the Cauchy problem in $L^{2}$ for first order system. A necessary condition is that the system must be uniformly diagonalizable, or equivalently that it admits a bounded symmetrizer. A sufficient condition is that it admits a smooth (Lipschtitz) symmetrizer, which is true when the system is hyperbolic, diagonalizable with eigenvalues of constant multiplicities. Counterexamples show that uniform diagonalizability is not sufficient in general for systems with variable coefficients and indicate that the symplectic properties of the set $\Sigma$ of the singular points of the characteristic variety are important. In this paper, give a new class of systems for which the Cauchy problem is well posed in $L^{2}$. The main assumption is that $\Sigma$ is a smooth involutive manifold and the system is transversally strictly hyperbolic.
\end{abstract}

\section{Introduction}

In this note, we give a new class of first order systems

$$
L\left(t, x, D_{t}, D_{x}\right)=D_{t}-\sum_{j=1}^{d} A_{j}(t, x) D_{x_{j}}=D_{t}-A\left(t, x, D_{x}\right)
$$

*Institut de Mathématiques de Bordeaux, UMR 5251: Guy.Metivier@math.ubordeaux.fr

${ }^{\dagger}$ Department of Mathematics, Graduate School of Science, Osaka University, Osaka: nishitani@math.sci.osaka-u.ac.jp 
which are strongly hyperbolic, which means by definition that for all lower order term $B$ the Cauchy problem for $L+B$ with initial data on the surface $\{t=0\}$ is well-posed in $C^{\infty}$. Here we use the notation $D=-i \partial$ for partial derivatives.

Strong hyperbolicity is satisfied as soon as the Cauchy problem is well posed in $L^{2}$ and satisfies resolvent estimates of the form

$$
\gamma\|u\|_{L^{2}} \lesssim\|(L+i \gamma \nu) u\|_{L^{2}}, \quad \text { for } \gamma \geq \gamma_{0},
$$

where $\nu$ denotes the conormal to the initial surface, or semi-group estimates of the form

$$
\|u(t)\|_{L^{2}} \lesssim\|u(0)\|+\int_{0}^{t}\left\|L u\left(t^{\prime}\right)\right\|_{L^{2}} d t^{\prime} .
$$

In these cases, additional zero-th order term are bounded in $L^{2}$ and absorbed by choosing $\gamma$ large or by Gronwall's lemma. Note that (1.3) implies (1.2).

A necessary condition for (1.2) is that the system $L\left(t, x, D_{t}, D_{x}\right)$ must be uniformly diagonalizable, which means that is there is a family of matrices $T(t, x, \xi)$, such that $T$ and $T^{-1}$ are uniformly bounded and $T^{-1} A T$ is diagonal. There are other equivalent formulations, for instance that there is a bounded family of symmetrizers, that is a family of hermitian symmetric matrices $S(t, x, \xi)$, such that $S$ and $S^{-1}$ are uniformly bounded and $S A$ is symmetric (see e.g [4]).

For systems with constant coefficients, strong hyperbolicity is equivalent to uniform diagonalizability (see [5], [14]). This is not true for general systems with variable coefficients. Even more, there are examples of uniformly diagonalizable systems for which the Cauchy problem is ill-posed in $C^{\infty}$ (and thus not strongly hyperbolic) (see $[4,13,7]$ ). However, strong hyperbolicity is known for diagonalizable systems with constant multiplicities, and more generally for systems which admit smooth symmetrizers $S(t, x, \xi)$. In [7], the required smoothness has been decreased to Lipschitz regularity in $(t, x, \xi)$ for $\xi \neq 0$. A necessary condition for $L$ to be strongly hyperbolic is that the geometric multiplicity of any multiple eigenvalue $\tau$ of $L(t, x, \tau, \xi)$ is not less than the half of the algebraic multiplicity +1 and strong hyperbolicity is known for a class of systems which generalizes single effectively hyperbolic operators and which are not symmetrizable (see $[11,12])$.

How to characterize strongly hyperbolic systems is an interesting problem which is not yet settled. This note brings a new piece to the understanding of this question. 
Before stating our main result, let us introduce some notations. We will work within the following framework.

Assumption 1.1. The coefficient matrices $A_{j}(t, x)$ are $C^{\infty}$ and constant outside a compact set. They act in a $N$-dimensional space denoted by $\mathbb{E}$. Moreover, for all $(t, x, \xi)$ the eigenvalues of $A(t, x, \xi)=\sum \xi_{j} A_{j}(t, x)$ are real and semi-simple.

In particular $L$ is hyperbolic in the time direction and diagonalizable. We denote by $\mathcal{C}$ the characteristic variety of $L$, that is the set of $(t, x, \tau, \xi) \in$ $T^{*} \mathbb{R}^{1+d} \backslash\{0\}$ such that $\operatorname{det} L(t, x, \tau, \xi)=0$. Note that Assumption 1.1 implies that at characteristic points,

$$
\operatorname{dim} \operatorname{Ker} L(t, x, \tau, \xi)=\text { multiplicity of the eigenvalue } \tau \text {. }
$$

If $\rho=(\underline{t}, \underline{x}, \underline{\tau}, \underline{\xi})$ is a regular point of $\mathcal{C}$, then Assumption 1.1 implies that on a neighborhood of $\rho, \mathcal{C}$ is given by an equation $\tau=\mu(t, x, \xi)$ and that $\mu$ is an eigenvalue of constant multiplicity of $A(t, x, \xi)$ for $(t, x, \xi)$ close to $(\underline{t}, \underline{x}, \underline{\xi})$ since $\mu(t, x, \xi)$ is the unique eigenvalue close to $\underline{\tau}$.

As mentioned above, variable multiplicities form an important obstacle to strong hyperbolicity and we have to impose conditions at singular points. Recall first the invariant definition of the localized system $L_{\rho}$ at $\rho \in \mathcal{C}$ :

$$
L_{\rho}(\dot{\rho})=\varpi_{\rho}\left(L^{\prime}(\rho) \cdot \dot{\rho}\right) \imath_{\rho}
$$

where $\imath_{\rho}$ is the injection of $\operatorname{ker} L(\rho)$ into $\mathbb{E}, \varpi_{\rho}$ is the projection from $\mathbb{E}$ onto $\mathbb{E} /$ range $L(\rho)$ and $L^{\prime}$ is the derivative of $L$. Because $\operatorname{ker} L(\rho) \cap \operatorname{range} L_{\rho}=$ $\{0\}$ by Assumption 1.1, $L_{\rho}$ can also be seen as a matrix with values in $\operatorname{Hom}(\operatorname{Ker} L(\rho))$. Recall that $L_{\rho}$ is hyperbolic in the time direction, and more generally, that the cone of hyperbolic directions of $L_{\rho}$ contains the cone of hyperbolic directions of $L$.

We make the following natural assumption on the set $\Sigma$ of singular points of $\mathcal{C}$.

Assumption 1.2. $\Sigma \subset \mathcal{C}$ is a smooth $C^{\infty}$ manifold in $T^{*} \mathbb{R}^{1+d} \backslash\{0\}$ and on each component of $\Sigma$ the dimension of $\operatorname{ker} L(\rho)$ is constant.

Lemma 1.3. When Assumptions 1.1 and 1.2 are satisfied, then $L_{\rho}(\dot{\rho})=0$ when $\rho \in \Sigma$ and $\dot{\rho} \in T_{\rho}(\Sigma)$. 
This lemma will be proved in Section 4. It implies that the symbol $L_{\rho}$ can be seen as defined on $T^{*} \mathbb{R}^{d+1} / T_{\rho} \Sigma$, that is on directions $\dot{\rho}$ which are transversal to $\Sigma$. Note that, because $L_{\rho}$ is hyperbolic in the direction $d t$, this direction is not characteristic for $L_{\rho}$ and thus transverse to $\Sigma$. The first natural case to consider is when $L_{\rho}$ is strictly hyperbolic in these transverse directions:

Assumption 1.4. For all $\rho \in \Sigma, L_{\rho}(\dot{\rho})$ is strictly hyperbolic in the time direction, on $T^{*} \mathbb{R}^{d+1} / T_{\rho} \Sigma$.

This condition is called linear splitting in [8]. In some sense, the three assumptions above describe the simplest and most regular structure for eigenvalue crossing along a variety $\Sigma$. However, these conditions are not sufficient to imply strong hyperbolicity. The counterexample in [7] and the analysis in [12] based on effectively hyperbolicity, indicate that the symplectic properties of the $\Sigma$ plays an important role. In particular, the counterexample in [7] is based on a reduction to the harmonic oscillator and this relies on the property that the symplectic form is not identically 0 on $T \Sigma$. In this paper, we go in the opposite direction and we can now formulate our main assumption.

Assumption 1.5. All $\rho \in \Sigma$ has a neighborhood on which one of the following condition is satisfied:

i) $\Sigma$ is of co-dimension two in $T^{*} \mathbb{R}^{1+d} \backslash\{0\}$,

ii) $\Sigma$ is of co-dimension three in $T^{*} \mathbb{R}^{1+d} \backslash\{0\}$ and $\operatorname{dim} \operatorname{ker} L(\rho)=2$,

iii) $\Sigma$ is an involutive submanifold of $T^{*} \mathbb{R}^{1+d} \backslash\{0\}$.

We can now state our main result.

Theorem 1.6. Consider a first order system L (1.1) satisfying the Assumptions 1.1, 1.2, 1.4 and 1.5. Then the Cauchy problem for $L$ with initial data on $\{t=0\}$ is well-posed in $L^{2}$. In particular $L$ is a strongly hyperbolic system.

Indeed, only the third case in Assumption 1.5 is new. In the first two cases, as noted in $[8,7,10]$, there is a smooth microlocal symmetrizer $S(t, x, \xi)$. This will be recalled in the proof of the theorem. On the contrary, in general, there is no smooth symmetrizer under the condition iii). Indeed, the Assumption 1.4 implies that there is a symmetrizer $S\left(t, x, \xi, \xi^{\prime}\right)$ which is homogeneous of degree 0 and smooth with respect to the transversal variables $\xi^{\prime} \neq 0$. Thus, under Assumptions 1.1, 1.2 and 1.4, the system is uniformly 
diagonalizable, but in general, the symmetrizer is singular at $\Sigma$. Because of this singularity, we cannot use the classical pseudo-differential calculus. But, using the precise description of the singularity, we can use instead a second microlocalization, and this is where the condition iii) comes in.

Theorem 1.6 is proved in Section 3. The main step is to obtain semigroup estimates of the form (1.3) on $[0, T] \times \mathbb{R}^{d}$. Since the assumptions are also satisfied by the adjoint system $L^{*}$, we have similar estimates for the backward Cauchy problem for $L^{*}$, and thus existence of weak solutions in $L^{2}$ for the direct problem; by Friedrich's Lemma they are strong solutions and satisfy the energy estimate; differentiating the equation, we see that they are as smooth as the data. The geometric formulation of the assumptions, imply that they remain satisfied after all space-like change of variables, implying local uniqueness, finite speed of propagation and local existence and uniqueness for the Cauchy problem.

The proof of the main $L^{2}$ estimate is microlocal. Near $(0, \underline{x}, \xi)$, one can perform a smooth reduction of the system into blocks $A_{k}$ associated to the distinct eigenvalues $\underline{\tau}_{k}$ of $A(0, \underline{x}, \underline{\xi})$. If $\rho_{k}=\left(0, \underline{x}, \underline{\tau}_{k}, \underline{\xi}\right)$ is a regular point of $\mathcal{C}$, then near $(0, \underline{x}, \underline{\xi}), A_{k}$ has a unique real eigenvalue and the estimate is well known. If $\rho_{k} \in \Sigma$, in cases $i$ ) and $i i$ ) there is a smooth symmetrizer and the $L^{2}$ microlocal energy estimate follows. In case $\left.i i i\right)$ we can reduce the problem to a model case of the following form:

$$
L_{\text {mod }}\left(t, x, D_{t}, D_{x}\right)=D_{t}-\sum_{j=1}^{l} A_{j}\left(t, x, D_{x}\right) D_{x_{j}}
$$

where the $A_{j}$ are classical pseudo-differential operators of order 0 . In this model, the involutive manifold is $\Sigma_{\text {mod }}=\left\{\tau=\xi_{1}=\ldots=\xi_{l}=0\right\}$. This model is analyzed in Section 5. The key is that, by Assumption 1.4 there is a symmetrizer $S\left(t, x, \xi, \xi^{\prime}\right)$ which can be quantified so that the error $\operatorname{Im}\left(L_{\text {mod }} S\right)$ is bounded in $L^{2}$. The reduction to the model case, is made using a Fourier integral operator which quantifies a canonical transformation which maps the involutive manifold $\Sigma$ to the model case $\Sigma_{\text {mod }}$. However, since we are considering the Cauchy problem, we need to preserve time in this reduction. Details are given in Section 4. 


\section{The microlocal symbolic analysis}

In preparation for the proof of Theorem 1.6, we analyze first the properties of the symbol $L(t, x, \tau, \xi)=\tau \operatorname{Id}-A(t, x, \xi)$. Let $(\underline{t}, \underline{x}, \xi) \in \mathbb{R}^{1+d} \times \mathbb{R}^{d} \backslash\{0\}$. We denote by $\underline{\tau}_{k}$, the distinct eigenvalues of $A(\underline{t}, \underline{x}, \underline{\xi})$ so that the points $\rho_{k}=\left(\underline{t}, \underline{x}, \underline{\tau}_{k}, \underline{\xi}\right)$ belong to the characteristic variety $\mathcal{C}$.

For $(t, x, \xi)$ in a small conical neighborhood $\omega$ of $(\underline{t}, \underline{x}, \underline{\xi})$, there is a smooth invertible matrix $P(t, x, \xi)$, homogeneous of degree 0 in $\bar{\xi}$, such that $P^{-1} A P$ is block diagonal

$$
P^{-1}(t, x, \xi) A(t, x, \xi) P(t, x, \xi)=\operatorname{diag}\left(A_{k}(t, x, \xi)\right)
$$

where the eigenvalues of the blocks $A_{k}$ are close to $\underline{\tau}_{k}$, and its dimension $r_{k}$ is the multiplicity of $\underline{\tau}_{k}$.

There are several cases.

1) If $\rho_{k}$ is a regular point of $\mathcal{C}$, locally near $\rho_{k}, \mathcal{C}$ is given by a smooth equation $\tau=\mu(t, x, \xi)$ and

$$
A_{k}(t, x, \xi)=\mu(t, x, \xi) \mathrm{Id} .
$$

2) If $\rho_{k} \in \Sigma$ is a singular point, we can write

$$
A_{k}(t, x, \xi)=\mu(t, x, \xi) \operatorname{Id}+A_{k}^{\prime}(t, x, \xi),
$$

where $A_{k}^{\prime}$ is traceless. By Assumption 1.2, near $\rho_{k}, \tau-\mu$ and $A^{\prime}$ vanish on $\Sigma$ and therefore; $\Sigma$ is given by the equations

$$
\tau=\mu(t, x, \xi), \quad q_{j}(x, \xi)=0, \quad j=1, \ldots, l .
$$

Therefore, $A$ has the form

$$
A_{k}(t, x, \xi)=\mu(t, x, \xi) \operatorname{Id}+\sum_{j=1}^{l} A_{k, j}(t, x, \xi) q_{j}(x, \xi) .
$$

The localized system at $\rho_{k}, L_{\rho_{k}}^{\prime}(\dot{\rho})$, is conjugated to

$$
(\dot{\tau}+\dot{\mu}) \mathrm{Id}+\sum A_{k, j} \dot{q}_{j}
$$

where $\dot{\mu}=\dot{t} \partial_{t} \mu+\dot{x} \partial_{x} \mu+\dot{\xi} \partial_{\xi} \mu$ and with similar definition for $\dot{q}_{j}$. This clearly proves Lemma 1.3. Because the $d q_{j}$ are independent, Assumption 1.4 implies, and indeed is equivalent to: 
Lemma 2.1. For all $\eta \in \mathbb{R}^{l} \backslash\{0\}$, the eigenvalues of $\sum \eta_{j} A_{k j}(\underline{t}, \underline{x}, \underline{\xi})$ are real and simple.

Proposition 2.2. There is a conical neighborhood $\omega$ of $(\underline{t}, \underline{x}, \underline{\xi})$ and there is a symmetric matrix $\mathcal{S}(t, x, \xi, \eta), C^{\infty}$ for $(t, x, \xi) \in \omega$, and $\eta \in \mathbb{R}^{l} \backslash\{0\}$, homogeneous of degree 0 both in $\xi$ and $\eta$, and such that $\mathcal{S}$ is positive definite, $\mathcal{S}^{-1}$ is bounded, and

$$
\operatorname{Im}\left(\mathcal{S}(t, x, \xi, q(x, \xi)) A_{k}(t, x, \xi)\right)=0
$$

Proof. (See [9] ) For $(t, x, \xi) \in \omega$ and $\eta \in \mathbb{R}^{l}$, introduce the symbol

$$
\mathcal{A}_{k}(t, x, \xi, \eta)=\sum_{j=1}^{l} \eta_{j} A_{k, j}(t, x, \xi)
$$

By the lemma, for $|\eta|=1$, the eigenvalues of $\mathcal{A}_{k}(\underline{t}, \underline{x}, \underline{\xi}, \eta)$ are simple. By perturbation, this property remains true for $(t, x, \xi)$ close to $(\underline{t}, \underline{x}, \underline{\xi})$, and therefore, shrinking $\omega$ if necessary, there is a smooth matrix $\mathcal{P}(t, x, \bar{\xi}, \eta)$ for $(t, x, \xi) \in \omega$ and $\eta \neq 0$, homogeneous of degree 0 in $\xi$ and in $\eta$, such that

$$
\mathcal{P} \mathcal{A}_{k} \mathcal{P}^{-1}=\operatorname{diag}\left(\beta_{1}, \ldots, \beta_{r_{k}}\right)
$$

is diagonal with entries $\beta_{j}(t, x, \xi, \eta)$. Then

$$
\mathcal{S}=\mathcal{P}^{*} \mathcal{P}
$$

is self adjoint and positive definite, and satisfies (2.6) since for $\eta=q(t, x, \xi)$ the eigenvalues of $\mathcal{A}_{k}(t, x, \xi, q(t, x, \xi))=A_{k}(t, x, \xi)$ are real.

We now discuss the consequences of Assumption 1.5.

Lemma 2.3. If property $i$ ) or $i i)$ of Assumption 1.5 is satisfied at $\rho_{k}$, there is a conical neighborhood $\omega$ of $(\underline{t}, \underline{x}, \underline{\xi})$ and there is a smooth positive definite matrix $S_{k}(t, x, \xi)$ for $(t, x, \xi) \in \omega$, homogeneous of degree 0 in $\xi$ and such that $S_{k} A_{k}$ is self adjoint.

Proof. In these cases we can find a smooth microlocal symmetrizer. For the explicit expression of the symmetrizers we refer to $[10,7]$. 
Proposition 2.4. If condition iii) of Assumption 1.5 is satisfied at $\rho_{k}$, there is a canonical transformation $\Psi=\rho \mapsto \tilde{\rho}$ in a neighborhood of $\rho_{k}$ such that $\tilde{t}=t$ and $\tilde{\Sigma}=\Psi(\Sigma)$ is given by the equations

$$
\tilde{\tau}=0, \quad \tilde{\xi}_{1}=\ldots=\tilde{\xi}_{l}=0 .
$$

Proof. We construct $\Psi$ in two steps. First we consider the flow of $H_{\tau-\mu}$, the Hamilton vector field of $\tau-\mu$,

$$
\Psi_{1}=\exp \left((t-\underline{t}) H_{\tau-\mu}\right): \rho \mapsto \rho_{1}
$$

It is a canonical transformation defined on a conical neighborhood of $\rho_{k}$, such that $t_{1}=t$ and which transforms $\tau-\mu$ into $\tau_{1}$. Therefore, $\Sigma_{1}$, the image of $\Sigma$ is given by equations

$$
\tau_{1}=0, \quad q_{1,1}=\ldots=q_{1, l}=0 .
$$

Since $\Sigma_{1}$ is involutive the Poisson brackets of these equations, and in particular $\partial_{t_{1}} q_{1, j}$, vanish on $\Sigma$. Because $\partial_{t_{1}} q_{1, j}$ is independent of $\tau_{1}$ and the $d_{x, \xi} \tilde{q}_{1, j}$ are linearly independent, this means that there are smooth homogenous functions $m_{j, k}$ such that

$$
\partial_{t_{1}} q_{1, j}=\partial_{t} g_{1, j}=\sum m_{j, k} q_{1, k}
$$

Denoting by $Q_{1}$ the vector with components $q_{1, j}$ and by $M$ the matrix with entries $m_{j, k}$, this means that $\partial_{t} Q_{1}=M Q_{1}$. Introduce $P(t, x, \xi)$ such that

$$
\partial_{t} P=-P M, \quad P_{\mid t=0}=\text { Id } .
$$

Hence $\partial_{t}\left(P Q_{1}\right)=0$ and $\tilde{Q}_{1}=P Q_{1}$ depends only on $(x, \xi)$. This shows that $\Sigma_{1}$ is given locally by the equations

$$
\tau_{1}=0, \quad \tilde{q}_{1, j}(x, \xi)=0 .
$$

In other words, near $\rho_{k}$,

$$
\Sigma_{1}=\left\{(t, x, 0, \xi) ;(x, \xi) \in \underline{\Sigma}_{1}\right\}
$$

where $\underline{\Sigma}_{1}$ is given by the equations $\tilde{q}_{1, j}(x, \xi)=0$. Moreover $\underline{\Sigma}_{1}$ is involutive in $T^{*} \mathbb{R}^{d}$ and there is a canonical transformation $\Psi_{2}$ in $T^{*} \mathbb{R}^{d}$ which transforms $\tilde{q}_{1, j}$ into $\xi_{j}$ for $j=1, \ldots, l$. We extend it to $T^{*} \mathbb{R}^{1+d}$, by leaving $\left(t_{1}, \tau_{1}\right)$ invariant, and the composed transformation $\Psi_{2} \circ \Psi_{1}$ has the desired properties. 


\section{Proof of Theorem 1.6}

As recalled in the introduction, it is sufficient to prove the following energy estimate:

Theorem 3.1. Under the assumptions of Theorem 1.6, there are $T>0$ and $C$ such that for all smooth function $u$, one has

$$
\|u(t)\|_{L^{2}} \leq C\|u(0)\|+C \int_{0}^{t}\left\|L u\left(t^{\prime}\right)\right\|_{L^{2}} d t^{\prime} .
$$

To prove this, we construct a finite partition of unity on $T^{*} \mathbb{R}^{d}$

$$
1=\chi_{\infty}(x)+\sum_{j=0}^{n} \chi_{j}(x, \xi)
$$

and prove the estimate (3.1) for each piece $u_{\infty}=\chi_{\infty} u$ and $u_{j}=\chi_{j}\left(x, D_{x}\right) u$ separately. Indeed,

$$
\left\|L u_{j}(t)\right\|_{L^{2}} \lesssim\|L u(t)\|_{L^{2}}+\|u(t)\|_{L^{2}}, \quad\left\|u_{j}(0)\right\|_{L^{2}} \lesssim\|u(0)\|_{L^{2}}
$$

so, adding the estimates for $u_{j}$ we get that

$$
\|u(t)\|_{L^{2}} \leq C\|u(0)\|+C \int_{0}^{t}\left\|L u\left(t^{\prime}\right)\right\|_{L^{2}}+C \int_{0}^{t}\left\|u\left(t^{\prime}\right)\right\|_{L^{2}} d t^{\prime},
$$

which, by Gronwall's lemma, implies (3.1).

We choose $\chi_{\infty}$ equal to 1 for $|x| \geq R$ with $R$ so large that $L=L_{\infty}=$ $D_{t}-A_{\infty}\left(D_{x}\right)$ has constant coefficients on the support of $\chi_{\infty}$. Our assumptions imply that $L$, thus $L_{\infty}$, is uniformly diagonalizable. Hence there is a bounded symmetrizer $S_{\infty}(\xi)$ for $A_{\infty}(\xi)$ and the $L^{2}$ estimate for $u_{\infty}$ follows, using the Fourier transform.

We choose $\chi_{0}$ supported in $|\xi| \leq 2$. Then $\left\|\partial_{t} \chi_{0} u\right\|_{L^{2}}=O\left(\|u\|_{L^{2}}+\|L u\|_{L^{2}}\right)$ and the estimate for $\chi_{0}\left(x, D_{x}\right) u$ immediately follows.

Thus by compactness of $\{|x| \leq R\} \times S^{d-1}$, we are reduced to prove the following estimate:

Proposition 3.2. Under the assumptions of Theorem 1.6, for all $(\underline{x}, \underline{\xi}) \in$ $T^{*} \mathbb{R}^{d} \backslash\{0\}$, there are $T>0$, a conical neighborhood $\omega$ of $(\underline{x}, \underline{\xi})$ such that for 
all pseudo-differential symbol of degree $0, \chi(x, \xi)$ supported in $\omega$, there is a constant $C$ such that for all smooth function $u$,

$$
\left\|\chi\left(x, D_{x}\right) u(t)\right\|_{L^{2}} \leq C\|u(0)\|+C \int_{0}^{t}\left(\left\|L u\left(t^{\prime}\right)\right\|_{L^{2}} d t^{\prime}+\left\|u\left(t^{\prime}\right)\right\|_{L^{2}}\right) d t^{\prime} .
$$

Proof. a) From now on, we fix $(\underline{x}, \xi) \in T^{*} \mathbb{R}^{d} \backslash\{0\}$. We convert the symbolic analysis of the previous section into a pseudo-differential calculus. Let $P(t, x, \xi)$ such that $(2.1)$ holds in a conical neighborhood $\omega$ of $(0, \underline{x}, \underline{\xi})$. Let $\chi$ be supported in $\omega^{\prime}=\omega \cap\{t=0\}$ and introduce $v=P^{-1}\left(t, x, D_{x}\right) \chi\left(x, D_{x}\right) u$ and its component $v_{k}$ which in the block corresponding decomposition above. Let

$$
L_{k} v_{k}:=D_{t} v_{k}-A_{k}\left(t, x, D_{x}\right) \tilde{\chi}\left(x, D_{x}\right) v_{k}
$$

where $\tilde{\chi}$ is supported in $\omega^{\prime}$ and equal to 1 on a neighborhood of the support of $\chi$. Because the commutators are bounded in $L^{2}$, one has

$$
\left\|L_{k} v_{k}(t)\right\|_{L^{2}} \lesssim\|L u(t)\|_{L^{2}}+\|u(t)\|_{L^{2}}
$$

We claim that it is sufficient to prove the following energy estimate for each $v_{k}$ separately:

$$
\left\|v_{k}(t)\right\|_{L^{2}} \leq C\left\|v_{k}(0)\right\|+C \int_{0}^{t}\left(\left\|L_{k} v_{k}\left(t^{\prime}\right)\right\|_{L^{2}} d t^{\prime}+\left\|v_{k}\left(t^{\prime}\right)\right\|_{L^{2}}\right) d t^{\prime} .
$$

Indeed, with (3.5), it implies that

$$
\|v(t)\|_{L^{2}} \leq C\|u(0)\|+C \int_{0}^{t}\left(\left\|L u\left(t^{\prime}\right)\right\|_{L^{2}} d t^{\prime}+\left\|u\left(t^{\prime}\right)\right\|_{L^{2}}\right) d t^{\prime} .
$$

Moreover, $\chi\left(x, D_{x}\right) u=(\tilde{\chi} P)\left(t, x, D_{x}\right) v+R u$ where $R$ is of degree -1 and hence

$$
\left\|\chi\left(x, D_{x}\right) u(t)\right\|_{L^{2}} \leq\|v(t)\|_{L^{2}}+\|u(t)\|_{H^{-1}} .
$$

Finally, we note that the errors in $H^{-1}$ are also controlled, using that

$$
\left\|\partial_{t} u(t)\right\|_{H^{-1}} \lesssim\|L u(t)\|_{L^{2}}+\|u(t)\|_{L^{2}}
$$

and

$$
\|u(t)\|_{H^{-1}} \leq\|u(0)\|_{H^{-1}}+\int_{0}^{t}\left\|\partial_{t} u\left(t^{\prime}\right)\right\|_{H^{-1}} d t^{\prime} .
$$

Therefore, it only remains to prove (3.6). 
b) If $\rho_{k}$ is a regular point in $\mathcal{C}$, or if $\rho_{k} \in \Sigma$ and one of the condition $i)$ or $i i)$ of Assumption 1.5 is satisfied, there is a symmetrizer $S_{k}(t, x, \xi)$ for $A_{k}$, in a possibly smaller neighborhood $\omega$. One can choose $\gamma$ such that the energy

$$
\operatorname{Re}\left(\tilde{\chi} S_{k}\left(t, x, D_{x}\right) v_{k}, v_{k}\right)_{L^{2}}+\gamma\left(\left(1+\left|D_{x}\right|^{2}\right)^{-1} v_{k}, v_{k}\right)_{L^{2}}
$$

is positive definite and equivalent to $\left\|v_{k}\right\|_{L^{2}}$. Differentiating in time and using the classical pseudo-differential calculus, one obtains (3.6).

c) If $\rho_{k} \in \Sigma$ and the condition iii) of Assumption 1.5 is satisfied, we know by Proposition 2.4 that there is a canonical transformation $\Psi$ on a neighborhood of $\rho_{k}$ such that the symbol of $L_{k}$ is transformed to

$$
\tilde{\tau} \mathrm{Id}+\sum_{j=1}^{l} \tilde{\xi}_{j} \tilde{A}_{j}(\tilde{t}, \tilde{x}, \tilde{\xi})
$$

revealing the model operator

$$
\tilde{L}=D_{\tilde{t}}-\sum_{j=1}^{l} \tilde{A}_{j}\left(\tilde{t}, \tilde{x}, D_{\tilde{x}}\right) D_{\tilde{x}_{j}} .
$$

In this case the estimate (3.6) follows from the following two results.

Proposition 3.3. There are $T>0$, a conical neighborhood $\omega$ of $(\underline{x}, \xi)$ and a smooth family of elliptic Fourier integral operators $F=F_{t}$, for $t \in[0, T]$, associated to the canonical transformation $\Psi$ and such that for all pseudodifferential symbol of degree $0, \chi(x, \xi)$ supported in $\omega$

$$
\left(F L_{k}-\tilde{L} F\right) \chi\left(x, D_{x}\right)
$$

is bounded in $L^{2}$, uniformly in time.

Proposition 3.4. If $\tilde{\chi}$ is supported in a small conical neighborhood of $(\underline{\tilde{x}}, \underline{\tilde{\xi}})$ where $\Psi\left(\rho_{k}\right)=(0, \underline{\tilde{x}}, 0, \underline{\tilde{\xi}})$, there is a constant $C$ such for all $w \in H^{1}([0, T] \times$ $\left.\mathbb{R}^{d}\right)$ satisfies

$$
\left\|\tilde{\chi}\left(\tilde{x}, D_{\tilde{x}}\right) w(t)\right\|_{L^{2}} \leq C\|w(0)\|_{L^{2}}+C \int_{0}^{t}\left\|\tilde{L} w\left(t^{\prime}\right)\right\|_{L^{2}} d t^{\prime}
$$


Indeed, decreasing the neighborhoods if necessary, we can assume that $\tilde{\chi}=1$ on the image of the support of $\chi$. We apply this estimate to $w=$ $F \chi\left(x, D_{x}\right) v_{k}$, noticing that, by (3.10) and the uniform boundedness of $F$ in $L^{2}$, one has

$$
\|\tilde{L} w(t)\|_{L^{2}} \lesssim\left\|L_{k} v_{k}(t)\right\|_{L^{2}}+\left\|v_{k}(t)\right\|_{L^{2}} .
$$

Moreover, by microlocal ellipticity of $F$ on the support of $\chi$, we have

$$
\left\|\chi\left(x, D_{x}\right) v_{k}(t)\right\|_{L^{2}} \leq\left\|\tilde{\chi}\left(\tilde{x}, D_{\tilde{x}}\right) w(t)\right\|_{L^{2}}+\left\|v_{k}(t)\right\|_{H^{-1}} .
$$

Since $\left\|\left(1-\chi\left(x, D_{x}\right)\right) v_{k}(t)\right\|_{L^{2}}$ is bounded by the right-hand side of (3.6), combining these estimates, and arguing as in (3.8) to absorb the $H^{-1}$ error, we obtain (3.6) in the case iii) of Assumption 1.5.

It remains to prove the two propositions above. This is done in the next two sections.

\section{Reduction to the model}

In this section, we prove Proposition 3.3. Recall that the canonical transformation $\Psi$ is the composed of $\Psi_{1}$ and $\Psi_{2}$. The first is defined, for $t \in[0, T]$, by (2.11). The symbol $\mu$ is defined on a small conical neighborhood $\omega$ of $(0, \underline{x}, \underline{\xi})$, for simplicity we modify it, so that it is defined on $[0, T] \times T^{*} \mathbb{R}^{d}$, so that is supported in $\omega$ and equal to $\mu$ in a smaller neighborhood $\omega^{\prime}$ with $|\xi| \geq 1$. Decreasing $T$ if necessary, consider the Fourier integral operator

$$
F_{1} u(t, x)=\int e^{i \varphi(t, x, \xi)} \hat{u}(t, \xi) d \xi
$$

where $\varphi(t, x, \xi)$ solves for $t \in[0, T]$ the eikonal equation

$$
\partial_{t} \varphi=\mu\left(t, x, \partial_{x} \varphi\right), \quad \varphi(0, x, \xi)=x \cdot \xi,
$$

(see $[6,2,3,1]$ ). It is associated to the canonical transformation $\Psi_{1}$. We can consider it also as a family of Fourier integral operators $F_{1, t}$ on $\mathbb{R}^{d}$, associated to canonical transformations $\Psi_{1, t}$, which are obviously defined from $\Psi_{1}$. Moreover, this operator is elliptic. Therefore, (see e.g. [1, 3])

Lemma 4.1. There is a constant $C$ such that for $u$ smooth, $v=F_{1} u$ satisfies

$$
\|v(t)\|_{L^{2}} \leq C\|u(t)\|_{L^{2}}
$$




$$
\|u(t)\|_{L^{2}} \leq C\|v(t)\|_{L^{2}}+C\|u(t)\|_{H^{-1}} .
$$

Moreover,

$$
\left\|D_{t} v(t)-F_{1}\left(D_{t}+\mu\left(t, x, D_{x}\right)\right) u(t)\right\|_{L^{2}} \leq C\|u(t)\|_{L^{2}}
$$

and if $a\left(t, x, D_{x}\right)$ is a pseudo-differential operator of order 1 , then

$$
\left\|b\left(t, x, D_{x}\right) v(t)-F_{1} a\left(t, x, D_{x}\right) u\right\|_{L^{2}} \leq C\|u(t)\|_{L^{2}}
$$

where

$$
b(t, x, \xi)=a\left(\Psi_{1, t}^{-1}(t, x, \xi)\right) .
$$

Let $\chi$ be supported in a small conical neighborhood of $(\underline{x}, \underline{\xi})$. The Lemma implies that $F_{1}$ transforms $L_{k}=D_{t}-A_{k}\left(t, x, D_{x}\right) \chi\left(x, D_{x}\right)$ into

$$
D_{t}-\sum A_{1, j}\left(t, x, D_{x}\right) q_{1, j}\left(t, x, D_{x}\right)+B\left(t, x, \partial_{x}\right)
$$

where $B(t)$ are uniformly bounded in $L^{2}$ and, using the notations introduced in (2.12), and the $q_{1, j}$ is the function deduced from $q_{j}$ by $\Psi_{1}$. We have shown that they are independent of $t$, and that there is a canonical transformation $\Psi_{2}$ which transforms them into $\xi_{j}$. Therefore there is an elliptic Fourier integral operator $F_{2}$ on $\mathbb{R}^{d}$, bounded in $L^{2}$, and such that

$$
\begin{gathered}
\left\|F_{2} q_{1, j}\left(x, D_{x}\right) \chi_{1}\left(x, D_{x}\right) v-D_{x_{j}} F_{2} \chi_{1}\left(x, D_{x}\right) v\right\|_{L^{2}} \lesssim\|v\|_{L^{2}} \\
\left\|\chi_{1}\left(x, D_{x}\right) v\right\|_{L^{2}} \lesssim\left\|F_{2} \chi_{1}\left(x, D_{x}\right) v\right\|_{L^{2}}+\left\|\chi_{1}\left(x, D_{x}\right) v\right\|_{H^{-1}}
\end{gathered}
$$

where $\chi_{1}$ is supported in a small conical neighborhood of $(\underline{x}, \underline{\xi})$ and one can assume that $\chi_{1}=1$ on the image of $\omega$ by $\Psi_{1}$ for $0 \leq t \leq T$. Here we note that

$$
\left\|\left(1-\chi_{1}(x, D)\right) F_{1} \chi(x, D) v\right\|_{H^{s}} \lesssim\|v\|_{H^{s-1}},
$$

for $s=0,1$ and for any $\chi$ supported in $\omega$.

Combining with the lemma above, we see that the composed operator $F_{2} \circ \chi_{1} F_{1}$ satisfies the properties listed in Proposition 3.3, which is now proved. 


\section{Analysis of the model system}

In this section we prove Proposition 3.4. The symbol of the model operator (5.1) has the form

$$
A(t, x, \xi)=\sum_{j=1}^{l} \xi_{j} A_{j}(t, x, \xi)
$$

with some $1 \leq l<d$ where the $A_{k}$ are classical pseudo-differential symbols of degree 0 , independent of $(t, x)$ for $(t, x)$ outside a compact set. The coefficients are defined on a neighborhood of $(\underline{x}, \underline{\xi})$, for convenience we extend the coefficients $A_{j}$ to $[0, T] \times T^{*} \mathbb{R}^{d}$ by multiplying them by some cut-off function $\chi(t, x, \xi)$. We still call $A_{j}$ these extended symbols.

Introduce

$$
\mathcal{A}(t, x, \xi, \eta)=\sum_{k=1}^{l} \eta_{k} A_{k}(t, x, \xi)
$$

By Proposition 2.2, we know that the following condition is satisfied.

Assumption 5.1. There is a symmetric matrix $\mathcal{S}(t, x, \xi, \eta), C^{\infty}$ in $(t, x)$ and constant outside a compact, homogeneous of degree 0 and $C^{\infty}$ both in $\xi \neq 0$ and $\eta \neq 0$, such that $\mathcal{S}$ is positive definite, $\mathcal{S}^{-1}$ is bounded, and $\mathcal{S}\left(t, x, \xi, \xi^{\prime}\right) \mathcal{A}(t, x, \xi)$ is symmetric, where $\xi^{\prime}=\left(\xi_{1}, \ldots, \xi_{l}\right)$.

Proposition 3.4 is a direct consequence of the following estimate.

Proposition 5.2. Under the Assumption 5.1, there is a constant $C$ such for all $u \in H^{1}\left([0, T] \times \mathbb{R}^{d}\right)$ satisfies

$$
\|u(t)\|_{L^{2}} \leq C\|u(0)\|_{L^{2}}+C \int_{0}^{t}\left\|L u\left(t^{\prime}\right)\right\|_{L^{2}} d t^{\prime} .
$$

To prove this estimate, we use a symbolic calculus where symbols of degree $(m, k)$ satisfy for all multi-indices $\alpha$ and $\beta$,

$$
\left|\partial_{x}^{\alpha} \partial_{\xi^{\prime}}^{\beta^{\prime}} \partial_{\xi^{\prime \prime}}^{\beta^{\prime \prime}} a\right| \leq C_{\alpha \beta}(1+|\xi|)^{m-\left|\beta^{\prime \prime}\right|}\left(1+\left|\xi^{\prime}\right|\right)^{k-\left|\beta^{\prime}\right|}
$$

where $\xi=\left(\xi^{\prime}, \xi^{\prime \prime}\right), \xi^{\prime}=\left(\xi_{1}, \ldots, \xi_{l}\right) \xi^{\prime \prime}=\left(\xi_{l+1}, \ldots, \xi_{d}\right)$, for some constant $C_{\alpha \beta}$ independent of $(x, \xi)$. The set of all such $a(x, \xi)$ is denoted by $S^{m, k}$ which is noting but $S(\mathrm{~m}, \mathrm{~g})$ with

$$
\mathrm{g}=|d x|^{2}+\frac{\left|d \xi^{\prime}\right|^{2}}{1+\left|\xi^{\prime}\right|^{2}}+\frac{\left|d \xi^{\prime \prime}\right|^{2}}{1+|\xi|^{2}}, \quad \mathrm{~m}=(1+|\xi|)^{m}\left(1+\left|\xi^{\prime}\right|\right)^{k}
$$


(see [3, Chapter XVIII] for the definition of $S(\mathrm{~m}, \mathrm{~g})$ and the associated pseudo-differential calculus). The key remark is that the symbol (5.1) belongs to $S^{0,1}$, so that the classical proof of the $L^{2}$ energy estimate works with symmetrizers in $S^{0,0}$, which is exactly the case of $\mathcal{S}\left(t, x, \xi, \xi^{\prime}\right)$. Let us proceed to the details.

We use Weyl's quantification op $(a)$ for symbols, but any other quantification would do since we consider only the principal terms. The rules of the symbolic calculus we need are collected in the following lemma, and follow from the general calculus developed in [3].

Lemma 5.3. i) Operators op $(a)$ with $a \in S^{0,0}$ are bounded in $L^{2}$.

ii) If $a \in S^{0,1}$ and $b \in S^{0,0}$ then $\mathrm{op}(a) \circ \mathrm{op}(b)-\mathrm{op}(a b)$ is bounded in $L^{2}$.

iii) If $S$ is a $n \times n$ self adjoint matrix of symbols in $S^{0,0}$, such that for all $(x, \xi) \in T^{*} \mathbb{R}^{d}$ with $\left|\xi^{\prime}\right| \geq 1$ and all vector $u \in \mathbb{C}^{n}$ one has

$$
c|u|^{2} \leq(S(x, \xi) u, u)
$$

for some $c>0$, then $\operatorname{op}(S)$ is self adjoint in $L^{2}\left(\mathbb{R}^{d} ; \mathbb{C}^{n}\right)$ and there is a constant $C$ such that for all $u \in L^{2}\left(\mathbb{R}^{d}\right)$

$$
\frac{c}{2}\|u\|_{L^{2}}^{2} \leq(\operatorname{op}(S) u, u)_{L^{2}}+C\left\|\left(1+\left|D_{x^{\prime}}\right|^{2}\right)^{-1 / 2} u\right\|_{L^{2}}^{2} .
$$

Moreover, the bounds are uniform if the symbols $(a, b, S)$ remains in bounded sets of the given classes $S^{0, m}$.

Proof of Proposition 3.4. Let $\chi_{0} \in C_{0}^{\infty}\left(\mathbb{R}^{l}\right)$ be supported in $|\eta|<2$ and such that $\chi_{0}(\eta)=1$ for $|\eta|<1$. Denote $\chi=1-\chi_{0}$. Introduce

$$
S_{0}(t, x, \xi)=\mathcal{S}\left(t, x, \xi, \xi^{\prime}\right) \chi\left(\xi^{\prime}\right)+\chi_{0}\left(\xi^{\prime}\right) .
$$

This is a self adjoint matrix of symbols in $S^{0,0}$, depending on the parameter $t$, and which satisfies (5.5). Thus, using (5.6), there is $\gamma>0$ and $c>0$ such that the operator op $(S(t))$ associated to the symbol

$$
S(t, x, \xi)=S_{0}\left(t, x, \xi, \xi^{\prime}\right)+\gamma\left(1+\left|\xi^{\prime}\right|^{2}\right)^{-1}
$$

satisfies for all $t \in[0, T]$ and $u \in L^{2}\left(\mathbb{R}^{d}\right)$

$$
c\|u\|_{L^{2}}^{2} \leq(\operatorname{op}(S(t)) u, u)_{L^{2}} .
$$


We fix $\gamma$ such that this property is satisfied. Using $i$ ) of the lemma, we see that there is another constant $C$ such that

$$
(\operatorname{op}(S(t)) u, u)_{L^{2}} \leq C\|u\|_{L^{2}}^{2} .
$$

Given a smooth $u \in C^{1}\left([0, T] ; H^{1}\left(\mathbb{R}^{d}\right)\right.$. Consider the energy

$$
E(t)=(\operatorname{op}(S(t)) u, u)_{L^{2}} \approx\|u(t)\|_{L^{2}}^{2} .
$$

The definition of $\operatorname{op}(S(t))$ shows that, for smooth functions,

$$
\left[\partial_{t}, \operatorname{op}(S(t))\right]=\operatorname{op}\left(\partial_{t} S(t)\right) .
$$

Moreover, $\partial_{t} u=i(L u+\mathrm{op}(A) u+B u)$, where $B$ is bounded in $L^{2}$. Therefore, dropping the parameter $t$ to simplify notations,

$$
\begin{aligned}
\partial_{t} E=-2 \operatorname{Im}(\operatorname{op}(S) L u, u)_{L^{2}}+\left(\operatorname{op}\left(\partial_{t} S\right) u, u\right)_{L^{2}} \\
-2 \operatorname{Im}(\operatorname{op}(S) \operatorname{op}(A) u, u)_{L^{2}}-2 \operatorname{Im}(\operatorname{op}(S) B u, u)_{L^{2}} .
\end{aligned}
$$

By the symbolic calculus, $\operatorname{op}\left(\partial_{t} S\right), \operatorname{op}(S) B$ and $\operatorname{op}(S) \operatorname{op}(A)-\operatorname{op}(S A)$ are bounded in $L^{2}$, uniformly in time. Because $\mathcal{S}$ symmetrizes $\mathcal{A}$, the definition of $S$ implies that

$$
\operatorname{Im}(S(t, x, \xi) A(t, x, \xi)) \in S^{0,0}
$$

and therefore $\operatorname{Im}(\mathrm{op}(S A))$ is bounded in $L^{2}$. This implies that the last three terms in (5.9) are $O\left(\|u\|_{L^{2}}\right)$. Integrating and using (5.7) and (5.8), yields

$$
\|u(t)\|_{L^{2}}^{2} \leq C\|u(0)\|_{L^{2}}^{2}+C \int_{0}^{t}\left(\left\|L u\left(t^{\prime}\right)\right\|_{L^{2}}\left\|u\left(t^{\prime}\right)\right\|_{L^{2}}+\left\|u\left(t^{\prime}\right)\right\|_{L^{2}}^{2}\right) d t^{\prime} .
$$

The estimate (5.3) follows for smooth functions. It extends to $u \in H^{1}$ by density.

Remark 5.4. The proof of the estimate (5.3) relies only on the existence of the symmetrizer $\mathcal{S}$ satisfying the properties listed in Lemma 5.3, not on strict hyperbolicity. 


\section{References}

[1] J.J.Duistermaat, Fourier integral operators, Progress in Mathematics, 130, Birkhäuser, 1994.

[2] Yu.V.Egorov, Canonical transformations and pseudodifferential operators, Trans. Moscow Math. Soc., 24 (1971) 3-28.

[3] L.Hörmandr, The Analysis of Linear Partial Differential Operators III, IV, Springer, Berlin, 1985.

[4] V.Ivrii and V.Petkov, Necessary conditions for the Cauchy problem for non strictly hyperbolic equations to be well posed. Uspehi Mat. Nauk, 29 (1974) 3-70.

[5] K.Kasahara and M.Yamaguti, Strongly hyperbolic systems of linear partial differential equations with constant coefficients, Mem. Coll. Sci. Univ. Kyoto. Ser. A. Math., 33 (1960/1961) 1-23.

[6] P.D.Lax, Asymptotic solutions of oscillatory initial value problems, Duke Math. J., 24 (1957) 627-646.

[7] G.Métivier, $L^{2}$ well posed Cauchy problems and symmetrizability, J. Ecole Polytechnique, 1 (2014) 39-70.

[8] G.Métivier and K.Zumbrum, Hyperbolic boundary value problems for symmetric systems with variable multiplicities, J. Diff. Equ., 211 (2005) 61-134.

[9] S.Mizohata, The theory of partial differential equations, Cambridge University Press, 1973.

[10] T.Nishitani, On strong hyperbolicity of systems, In: Hyperbolic equations, 102-114, Pitman Res. Notes Math. Ser., 158, Longman Sci. Tech., Harlow, 1987.

[11] T.Nishitani, Necessary conditions for strong hyperbolicity of first order systems, J. Anal. Math., 61 (1993) 181-229.

[12] T.Nishitani, Strongly hyperbolic systems of maximal rank, Publ. Res. Inst. Math. Sci., 33 (1997) 765-773. 
[13] G.Strang, Necessary and insufficient conditions for the well-posed Cauchy problems, J. Diff. Equ., 2 (1966) 107-114.

[14] G.Strang, On strong hyperbolicity, J. Kyoto Univ., 6 (1967) 397-417. 